Received: 05.02.2019

Revised: 01.03.2019

Accepted: 22.03 .2019

DOI: $10.17804 / 2410-9908.2019 .2 .057-070$

\title{
VORTEX FLOWS OF A VISCOUS INCOMPRESSIBLE FLUID AT CONSTANT VERTICAL VELOCITY UNDER PERFECT SLIP CONDITIONS
}

\author{
V. V. Privalova ${ }^{\text {a)* }}$ and E. Yu. Prosviryakov ${ }^{\text {b) }}$ \\ Institute of Engineering Science, Ural Branch of the Russian Academy of Sciences, \\ 34 Komsomolskaya St., Ekaterinburg, 620049, Russian Federation \\ a) (iD https://orcid.org/0000-0002-8648-0900 valentprival@ gmail.com; \\ b) (iD https://orcid.org/0000-0002-2349-7801 @ evgen_pros@mail.ru \\ "Corresponding author. E-mail: valentprival@gmail.com \\ Address for correspondence: 34 Komsomolskaya St., Ekaterinburg, 620049, Russian Federation \\ Tel.: +7 (343) 37535 76; fax: +7 (343) 3745330
}

In this paper, we study exact solutions of shear flows for a viscous incompressible fluid. The proposed solutions for the velocity components are linear functions of the longitudinal coordinates. Such solutions belong to the class of Lin-Sidorov-Aristov solutions for isobaric and isothermal processes. The obtained exact solution of the Navier-Stokes equation describes a new mechanism of momentum transfer in a medium and the flow of a vertically whirling fluid. A vertical twist in a fluid arises due to the allowance for inertial forces and a nonuniform distribution of velocities at the free boundary of the fluid layer. This solution allows us to describe the counterflow of incompressible fluid in a thin layer. The condition of perfect slip on the lower solid surface of the fluid layer is considered for the obtained exact general solution. The existence of points at which the velocity field vanishes inside the fluid layer is shown. It determines the existence of stagnant points and counterflows in the fluid.

Keywords: exact solution, vertical vortex, counterflow, stagnation point, perfect slip.

\section{References}

1. Ovsyannikov L.V. Gruppovoy analiz differentsialnykh uravneniy [Group Analysis of Differential Equations]. Moscow, Nauka Publ., 1978, 400 p. (In Russian).

2. Andreev V.K., Khapzov O.V., Pukhnachev V.V., Rodionov A.A. Primenenie teoretikogruppovykh metodov v gidrodinamike [Application of Group-Theoretical Methods in Hydrodynamics]. Novosibirsk, Nauka Publ., 1994, 320 p. (In Russian).

3. Aristov S.N., Knyazev D.V., Polyanin A.D. Exact solutions of the Navier-Stokes equations with the linear dependence of velocity components on two space variables. Theor. Found. Chem. Eng., 2009, vol. 43, no. 5, pp. 642-662. DOI: 10.1134/S0040579509050066.

4. Aristov S.N., Prosviryakov E.Yu. Inhomogeneous Couette Flows. Nelin. Dyn., 2014, vol. 10, no. 2, pp. 177-182. DOI: 10.20537/nd1402004. (In Russian).

5. Aristov S.N., Prosviryakov E.Yu. Stokes waves in vortical fluid. Nelin. Dyn., 2014, vol. 10, no. 3, pp. 309-318. DOI: 10.20537/nd1403005. (In Russian).

6. Aristov S.N., Prosviryakov E.Yu. A new class of exact solutions for three-dimensional thermal diffusion equations. Theor. Found. Chem. Eng., 2016, vol. 50, no. 3, pp. 286-293. DOI: $10.1134 / \mathrm{S} 0040579516030027$. 
7. Prosviryakov E.Yu. New Class of Exact Solutions of Navier-Stokes Equations with Exponential Dependence of Velocity on Two Spatial Coordinates. Theor. Found. Chem. Eng., 2019, vol. 53, no. 1, pp. 107-114. DOI: 10.1134/S0040579518060088.

8. Lin C.C. Note on a class of exact solutions in magneto-hydrodynamics. Arch. Rational Mech. Anal., 1957, vol. 1, pp. 391-395. DOI: 10.1007/BF00298016.

9. Sidorov A.F. One class of solutions of equations of gas dynamics and natural convection. In: Numerical and Analytical Methods of Solving Problems of Mechanics of Continuous Media (collected scientific papers), eds. A. F. Sidorov, Yu. N. Kondyurin, UNTs, AN SSSR Publ., Sverdlovsk, 1981, pp. 101-117. (In Russian).

10. Sidorov A.F. Two classes of solutions of the fluid and gas mechanics equations and their connection to traveling wave theory. J. Appl. Mech. Tech. Phys., 1989, vol. 30, no. 2, pp. 197-203. DOI: $10.1007 / \mathrm{BF} 00852164$.

11. Aristov S.N., Shvarts K.G. Vikhrevye techeniya advektivnoy prirody vo vrashchayushchemsya sloe zhidkosti [Vortex Flows of Advective Nature in Rotating Liquid Layer]. Perm, Perm State Univ. Publ., 2006. (In Russian).

12. Aristov S.N., Shvarts K.G. Vikhrevye Techeniya v Tonkikh Sloyakh Zhidkosti [Vortical Flows in Thin Fluid Layers]. Kirov, Vyatka State Univ. Publ., 2011, 207 p. (In Russian).

13. Andreev V.K. Resheniya Birikha uravneniy konvektsii i nekotorye ego obobshcheniya: Preprint no. 1-10 [Birikh Solutions to Convection Equations and Some of its Extensions]. IVM SO RAN Publ., Krasnoyarsk, 2010, 68 p. (In Russian).

14. Andreev V.K., Bekezhanova V.B. Stability of nonisothermal fluids (Review). J. Appl. Mech. Tech. Phys., 2013, vol. 54, no. 2, pp. 171-184. DOI: 10.1134/S0021894413020016.

15. Andreev V.K., Stepanova I.V. Unidirectional flows of binary mixtures within the framework of the Oberbeck-Boussinesq model. Fluid Dyn., 2016, vol. 51, no. 2, pp. 136-147. DOI: $10.1134 / \mathrm{S} 0015462816020022$.

16. Goncharova O.N., Hennenberg M., Rezanova E.V., Kabov O.A. Modening of the convective fluid flows with evaporation in the two-layer system. Interfacial Phenomena and Heat Transfer, 2013, vol. 1, no. 4, pp. 317-338. DOI: 10.1615/InterfacPhenomHeatTransfer.v1.i4.20.

17. Aristov S.N., Knyazev D.V., Polyanin A.D. Exact solutions of the Navier-Stokes equations with the linear dependence of velocity components on two space variables. Theor. Found. Chem. Eng., 2009, vol. 43, no. 5, pp. 642-662. DOI: 10.1134/S0040579509050066.

18. Betyaev V.K. Asimptoticheskie metody klassicheskoy dinamiki zhidkosti [Asymptotic Methods of Classical Fluid Dynamics]. Izhevsk, Institut Kompyuternykh Issledovaniy Publ., 2014, 516 p. (In Russian).

19. Andreev V.K. Influence of the interfacial internal energy on the thermocapillary steady flow. Journal of Siberian Federal University. Mathematics \& Physics, 2017, vol. 10, no. 4, pp. 537-547. DOI: 10.17516/1997-1397-2017-10-4-537-547.

20. Andreev V.K., Bekezhanova V.B., Efimova M.V., Ryzhkov I.I., Stepanova I.V. Nonclassical convection models: exact solutions and their stability. Computational Technologies, 2009, vol. 14 , no. 6 , pp. 5-18.

21. Bekezhanova V.B., Shefer I.A., Goncharova O.N., Rezanova E.B. Stability of two-layer fluid flows with evaporation at the interface. Fluid Dynamics, 2017, vol. 52, no. 2, pp. 189-200. DOI: $10.1134 / \mathrm{S} 001546281702003 \mathrm{X}$.

22. Goncharova O.N., Rezanova E.V., Lyulin Y.V., Kabov O.A. Analysis of a convective fluid flow with a concurrent gas flow with allowance for evaporation. High Temperature, 2017, vol. 55, no. 6, pp. 887-897. DOI: 10.1134/S0018151X17060074.

23. Aristov S.N., Prosviryakov E.Y. Large-scale flows of viscous incompressible vortical fluid. Russian Aeronautics (Iz. VUZ), 2015, vol. 58, no. 4, pp. 413-418. DOI: 10.3103/S1068799815040091.

24. Aristov S.N., Prosviryakov E.Y. Unsteady layered vertical fluid flows. Fluid Dynamics, 2016, vol. 51, no. 2, pp. 148-154. DOI: 10.1134/S0040579516030027. 
25. Aristov S.N., Privalova V.V., Prosviryakov E.Yu. Stationary nonisothermal Couette flow. Quadratic heating of the upper boundary of the fluid layer. Nonlin. Dyn., 2016, vol. 12, no. 2, pp. 167-178. DOI: 10.20537/nd1602001. (In Russian).

26. Privalova V.V., Prosviryakov E.Yu. Steady convective Coutte flow for quadratic heating of the lower boundary fluid layer. Nonlin. Dyn., 2018, vol. 14, no. 1, pp. 69-79. DOI: 10.20537/nd1801007. (In Russian).

27. Privalova V.V., Prosviryakov E.Yu. Couette-Hiemenz exact solutions for the steady creeping convective flow of a viscous incompressible fluid, with allowance made for heat recovery. Vestn. Samar. Gos. Tekhn. Univ., Ser. Fiz.-Mat. Nauki, 2018, vol. 22, no. 3, pp. 532-548. DOI: https://doi.org/10.14498/vsgtu1638. (In Russian).

28. Vinogradova O.I., Belyaev A.V. Wetting, roughness and flow boundary conditions. J. Phys.: Condens. Matter, 2011, vol. 23, p. 184104. DOI: 10.1088/0953-8984/23/18/184104.

29. Stone H., Stroock A., Ajdari A. Engineering flows in small devices: Microfluidics toward a lab-on-a-chip. Annual Review of Fluid Mechanics, 2004, vol. 36, pp. 381-411. DOI: 10.1146/annurev.fluid.36.050802.122124.

30. Quere D. Wetting and roughness. Annual Review of Materials Research, 2008, vol. 38, pp. 71-99. DOI: 10.1146/annurev.matsci.38.060407.132434.

31. Whitesides G.M. The origins and the future of microfluidics. Nature, 2006, vol. 442, pp. 368-373. DOI: 10.1038/nature05058.

32. Belyaev A.V., Vinogradova O.I. Effective slip in pressure-driven flow past superhydrophobic stripes. J. Fluid Mech., 2010, vol. 652, pp. 489-499. DOI: 10.1017/S0022112010000741.

33. Landau L.D., Lifshitz E.M. Fluid Mechanics. Pergamon Press, Oxford, 1987.

34. Pedlosky J. Geophysical Fluid Dynamics. Springer-Verlag, New York Inc., 1982.

35. Vinogradova O.I. Drainage of a thin liquid film confined between hydrophobic surfaces. Langmuir, 1995, vol. 11, iss. 6, pp. 2213-2220. DOI: 10.1021/la00006a059.

36. Mehdizadeh A., Oberlack M. Analytical and numerical investigations of laminar and turbulent Poiseuille-Ekman flow at different rotation rates. Physics of Fluids, vol. 22, no. 10, pp. 105104. DOI: $10.1063 / 1.3488039$.

37. Korotaev G.K., Mikhailova E.N., Shapiro N.B. Teoriya ekvatorialnykh protivotecheniy $v$ Mirovom okeane [Theory for the Equatorial Countercurrents in the World's Ocean]. Kiev, Naukova Dumka Publ., 1986. (In Russian). 
Подана в журнал: 05.02.2019

УДК 532.51

DOI: $10.17804 / 2410-9908.2019 .2 .057-070$

\title{
ВИХРЕВЫЕ ТЕЧЕНИЯ ВЯЗКОЙ НЕСЖИМАЕМОЙ ЖИДКОСТИ С УЧЕТОМ ПОСТОЯННОЙ ВЕРТИКАЛЬНОЙ СКОРОСТИ И УСЛОВИЯ ИДЕАЛЬНОГО СКОЛЬЖЕНИЯ
}

\author{
В. В. Привалова ${ }^{\text {a)* }}$, Е. Ю. Просвиряков ${ }^{\text {б) }}$ \\ Институт машиноведения Уральского отделения Российской академии наук, \\ ул. Комсомольская, 34, Екатеринбург, Российская Федераиия \\ a) (ID https://orcid.org/0000-0002-8648-0900 valentprival @gmail.com; \\ б) (iD https://orcid.org/0000-0002-2349-7801 @ evgen_pros@mail.ru \\ "Ответственный автор. Электронная почта: valentprival@gmail.com \\ Адрес для переписки: ул. Комсомольская, 34, Екатеринбург, Российская Федерация \\ Тел.: +7 (343) 375-35-76; факс: +7 (343) 374-53-30
}

В работе исследуются точные решения сдвиговых течений вязкой несжимаемой жидкости. Предложенные решения для компонент скоростей представляют собой линейные функции продольных координат. Такие решения относятся к классу решений Линя-Сидорова-Аристова для изобарических и изотермических процессов. Полученное точное решение уравнения Навье-Стокса описывает новый механизм переноса импульса в сплошной среде - течение вертикально завихренной жидкости без учета вращения. Вертикальная закрутка в жидкости возникает из-за учета сил инерции и неоднородного распределения скоростей на свободной границе слоя жидкости. Это решение позволяет описывать противотечения несжимаемой жидкости в тонком слое. Для полученного точного общего решения было рассмотрено условие идеального скольжения на нижней твердой поверхности слоя жидкости. Показано существование точек, в которых поле скоростей обращается в нуль внутри слоя жидкости, что определяет существование застойных точек и противотечений в жидкости.

Ключевые слова: точное решение, вертикальное завихрение, противотечение, застойная точка, идеальное скольжение.

\section{1. Введение}

Одним из важнейших уравнений в гидродинамике, применяющихся при математическом моделировании многих природных явлений и технических задач, являются уравнения Навье-Стокса. Нахождение точного общего решения уравнения Навье-Стокса для различных потоков осложняется тем, что оно нелинейное и сильно зависит от начальных и граничных условий. Проблема описания механизма переноса импульса в жидкости остается нерешенной в общем случае. Известно несколько методик нахождения точных решений, таких как групповой анализ $[1,2]$ и классы точных решений [3-7]. Под классом решений подразумевается специальная структура гидродинамических полей, определяющаяся непосредственно уравнениями Навье-Стокса.

Важную роль при исследовании изотермических течений вязких несжимаемых жидкостей сыграл класс точных решений, характеризующийся линейной зависимостью скоростей от продольных координат, описывающих поток. Этот класс решений впервые был описан Линем применительно к решению задач магнитной гидродинамики [8]. В дальнейшем этот класс решений был применен для решений задач естественной конвекции, теории бегущих волн, вихревых течений адвективного и конвективного характера и др. [9-15] Многие известные решения уравнения Навье-Стокса относятся именно к этому классу. Интерес 
к использованию этой методики нахождения точных решений уравнения Навье-Стокса продолжает расти и в настоящее время [16-22].

Благодаря этому классу можно построить точные решения уравнений Навье-Стокса [4, 5, 23-27], которые описывают появление вихрей в жидкости и образование противотечений в изотермических потоках. Анализ точных решений позволяет определять расслоение гидродинамических полей в рассматриваемом слое жидкости, в частности условия существования противотечений и усиление скоростей в горизонтальном направлении.

При выборе граничных условий на твердой поверхности течения жидкости в предложенной работе была рассмотрена модель идеального скольжения. Такой выбор обусловлен тем, что в последнее время в области механики жидкости и газа возникают вопросы о возможном большом эффективном скольжении жидкости по поверхностям, называемым супергидрофобными. На таких поверхностях предполагается значительное снижение сопротивления течения. Такие возможности делают супергидрофобные поверхности крайне перспективными для использования в микрофлюидике [28-32] - области науки, затрагивающей различные разделы химического синтеза и биологического анализа и основанной на управляемом течении жидкости в очень тонких каналах.

В настоящей работе предложено точное решение краевой задачи изотермического вихревого сдвигового течения вязкой несжимаемой жидкости в горизонтальном слое с проницаемыми границами и заданием условия идеального скольжения на твердой поверхности.

\section{2. Постановка задачи}

Для описания рассматриваемого процесса записываем уравнение Навье-Стокса для установившегося движения вязкой жидкости и уравнение несжимаемости. Уравнения спроецированы на оси прямоугольной декартовой системы координат. Стационарная система нелинейных уравнений в частных производных имеет следующий вид [33]:

$$
\begin{gathered}
V_{x} \frac{\partial V_{x}}{\partial x}+V_{y} \frac{\partial V_{x}}{\partial y}+V_{z} \frac{\partial V_{x}}{\partial z}=-\frac{\partial P}{\partial x}+v\left(\frac{\partial^{2} V_{x}}{\partial x^{2}}+\frac{\partial^{2} V_{x}}{\partial y^{2}}+\frac{\partial^{2} V_{x}}{\partial z^{2}}\right) \\
V_{x} \frac{\partial V_{y}}{\partial x}+V_{y} \frac{\partial V_{y}}{\partial y}+V_{z} \frac{\partial V_{y}}{\partial z}=-\frac{\partial P}{\partial y}+v\left(\frac{\partial^{2} V_{y}}{\partial x^{2}}+\frac{\partial^{2} V_{y}}{\partial y^{2}}+\frac{\partial^{2} V_{y}}{\partial z^{2}}\right) \\
V_{x} \frac{\partial V_{z}}{\partial x}+V_{y} \frac{\partial V_{z}}{\partial y}+V_{z} \frac{\partial V_{z}}{\partial z}=-\frac{\partial P}{\partial z}+v\left(\frac{\partial^{2} V_{z}}{\partial x^{2}}+\frac{\partial^{2} V_{z}}{\partial y^{2}}+\frac{\partial^{2} V_{z}}{\partial z^{2}}\right) ; \\
\frac{\partial V_{x}}{\partial x}+\frac{\partial V_{y}}{\partial y}+\frac{\partial V_{z}}{\partial z}=0 .
\end{gathered}
$$

Здесь в $V_{x}, V_{y}, V_{z}-$ компоненты вектора скорости; $P$ - отклонение давления от гидростатического, отнесенное к постоянной средней плотности $\rho ; v-$ кинематическая (молекулярная или турбулентная) вязкость [34]. Точное решение системы (1) будем искать в виде $[4,5,23,24]$ :

$$
\begin{gathered}
V_{x}=U(z)+y u(z) ; \\
V_{y}=V(z) ; \\
V_{z}=w(z) ;
\end{gathered}
$$




$$
P=P_{0}(z)
$$

Подставляем класс точных решений (2) в нелинейную систему (1). Система уравнений приобретает следующий вид:

$$
\begin{gathered}
V u+w\left(\frac{\partial U}{\partial z}+\frac{\partial u}{\partial z} y\right)=v\left(\frac{\partial^{2} U}{\partial z^{2}}+\frac{\partial^{2} u}{\partial z^{2}} y\right) \\
w \frac{\partial V}{\partial z}=v \frac{\partial^{2} V}{\partial z^{2}} \\
w \frac{\partial w}{\partial z}=-\frac{\partial P_{0}}{\partial z}+v \frac{\partial^{2} w}{\partial z^{2}} \\
\frac{\partial w}{\partial z}=0
\end{gathered}
$$

Из полученных выражений следует, что при таком выборе вида решений (2) вертикальная компонента скорости и давление являются постоянными функциями, заданными, например, на границе слоя жидкости. Можно принять давление равным атмосферному, заданному на верхней свободной поверхности. Таким образом, в системе (3) автоматически удовлетворяются последние два уравнения, определяемые граничными условиями. Решаем далее только первые два уравнения системы (3). Проведя преобразования в полученной системе (3), получаем выражения вида:

$$
A_{k}+B_{k} y=0
$$

Здесь $k=\overline{1,4}$ - номер уравнения. Уравнения при $k=1, k=2$ и $k=3$ - уравнения распространения импульсов, спроецированные на осях $O x, O y, O z$ соответственно; при $k=4$ - уравнение несжимаемости. Получаем коэффициенты следующего вида:

$$
\begin{gathered}
A_{1}=v U^{\prime \prime}-w U^{\prime}-V u ; \\
B_{1}=v u^{\prime \prime}-w u^{\prime} ; \\
A_{2}=v V^{\prime \prime}-w V^{\prime} .
\end{gathered}
$$

Приравниваем нулю коэффициент $B_{1}$ при переменной $y$ и свободные члены $A_{1}, A_{2}$ в полиномиальных выражениях (3). Обозначаем частные производные в уравнениях системы (3) штрихом, так как все искомые функции зависят только от переменной $z$. Получаем следующую систему, состоящую из трех нелинейных уравнений, для определения трех неизвестных функций:

$$
\begin{gathered}
v U^{\prime \prime}-w U^{\prime}-V u=0 ; \\
v u^{\prime \prime}-w u^{\prime}=0 ; \\
v V^{\prime \prime}-w V^{\prime}=0 .
\end{gathered}
$$




\section{3. Общее решение системы уравнений}

Приводим систему полученных уравнений (4) к безразмерному виду. Для этого выбираем следующие масштабные переменные: горизонтальные координаты $x$ и $y$ характеризуются масштабом $l$, а вертикальная координата $z$ - толщиной слоя жидкости $h$. Масштаб горизонтальных скоростей $V_{x}$ и $V_{y}$ обозначим [U]. Условие введения двух масштабных переменных длины существенно расширяет классические методы приведения к безразмерным переменным.

Далее безразмерные переменные $x, y$ и $z$ обозначаем теми же буквами. После приведения системы (4) к безразмерному виду уравнения принимают следующий вид:

$$
\begin{gathered}
U^{\prime \prime}-\operatorname{Re}_{w} U^{\prime}-\delta^{2} \operatorname{Re}_{U} V u=0 ; \\
u^{\prime \prime}-\operatorname{Re}_{w} u^{\prime}=0 ; \\
V^{\prime \prime}-\operatorname{Re}_{w} V^{\prime}=0 .
\end{gathered}
$$

Здесь $\operatorname{Re}_{U}=\frac{[U] l}{v}, \operatorname{Re}_{w}=\frac{w h}{v}-$ числа Рейнольдса, нижний индекс у которых обозначает, относительно какой скорости введен безразмерный комплекс; $\delta=\frac{h}{l}$ отношение вертикального и горизонтального характерных размеров.

Заметим, что система уравнений (5)-(7) является слабосвязанной, поскольку скорость $U$ вычисляется из уравнения (5) после интегрирования изолированных уравнений (6) и (7) для определения функции $u$ и $V$ соответственно.

Общее решение системы уравнений (5)-(7), записанное в порядке интегрирования, имеет вид:

$$
\begin{gathered}
u=\frac{\exp \left(\operatorname{Re}_{w} z\right)}{\operatorname{Re}_{w}} C_{1}+C_{2} ; \\
V=\frac{\exp \left(\operatorname{Re}_{w} z\right)}{\operatorname{Re}_{w}} C_{3}+C_{4} ; \\
U=\frac{\operatorname{Re}_{U} \delta^{2}}{2 \operatorname{Re}_{w}^{4}} C_{1} C_{3} \exp \left(2 \operatorname{Re}_{w} z\right)-\frac{\operatorname{Re}_{U} \delta^{2}}{\operatorname{Re}_{w}} C_{2} C_{4} z+ \\
+\frac{\exp \left(\operatorname{Re}_{w} z\right)}{\operatorname{Re}_{w}^{3}} \operatorname{Re}_{U} \delta^{2}\left(\operatorname{Re}_{w} z-1\right)\left(C_{2} C_{3}+C_{1} C_{4}\right)+\frac{\exp \left(\operatorname{Re}_{w} z\right)}{\operatorname{Re}_{w}} C_{5}+C_{6} .
\end{gathered}
$$

\section{4. Краевая задача с условием идеального скольжения на нижней границе слоя жидкости}

Рассмотрим частный случай краевой задачи для полученного общего решения (8) системы уравнений (5)-(7) с условием идеального скольжения на нижней твердой границе слоя жидкости. Краевая задача описывает течение вязкой несжимаемой жидкости в бесконечно протяженном горизонтальном слое толщиной $h$ в размерных переменных. 
При выполнении условия идеального скольжения на нижней границе компоненты скорости удовлетворяют следующим тождествам [33, 35]:

$$
\begin{aligned}
& \left.\frac{\partial V_{x}}{\partial z}\right|_{z=0}=0 ; \\
& \left.\frac{\partial V_{y}}{\partial z}\right|_{z=0}=0 .
\end{aligned}
$$

На верхней границе задаем компоненты скоростей следующим образом [4]:

$$
\begin{gathered}
V_{x}(1)=\cos \varphi+\frac{\mathrm{Ta}}{2 \operatorname{Re}_{U}} y ; \\
V_{y}(1)=\sin \varphi .
\end{gathered}
$$

Здесь $\varphi$ - произвольный угол; Та $=2 \Omega l^{2} / v-$ модифицированное число Тейлора [36]; $\Omega$ вертикальная компонента завихренности. Подставляем вид решений (2) в граничные условия (9) и (10). Таким образом, граничные условия на нижней границе, задаваемой уравнением плоскости $z=0$, имеют вид:

$$
\begin{aligned}
& \left.\frac{\partial U}{\partial z}\right|_{z=0}=0 ; \\
& \left.\frac{\partial u}{\partial z}\right|_{z=0}=0 ; \\
& \left.\frac{\partial V}{\partial z}\right|_{z=0}=0 .
\end{aligned}
$$

На верхней границе $z=1$ выполняются следующие условия, характеризующие параболический ветер [37] на свободной поверхности слоя жидкости:

$$
\begin{aligned}
& U(1)=\cos \varphi \\
& u(1)=\frac{\mathrm{Ta}}{2 \operatorname{Re}_{U}} \\
& V(1)=\sin \varphi
\end{aligned}
$$

Значения постоянных интегрирования вычисляются при подстановке краевых условий (11), (12) в общее решение (8):

$$
\begin{gathered}
C_{1}=0 ; \quad C_{2}=\frac{\mathrm{Ta}}{2 \operatorname{Re}_{U}} ; \quad C_{3}=0 ; \quad C_{4}=\sin \varphi ; \\
C_{5}=\frac{\delta^{2} \operatorname{Ta} \sin \varphi}{2 \operatorname{Re}_{w}} ; \quad C_{6}=\cos \varphi+\frac{\delta^{2} \operatorname{Ta} \sin \varphi}{2 \operatorname{Re}_{w}^{2}}\left[\operatorname{Re}_{w}-\exp \left(\operatorname{Re}_{w}\right)\right] .
\end{gathered}
$$


Подставляем полученные постоянные интегрирования (13) в общее решение (8) и получаем частное решение краевой задачи (5)-(7), (11), (12)

$$
\begin{gathered}
u=\frac{\mathrm{Ta}}{2 \mathrm{Re}_{U}} ; \\
V=\sin \varphi \\
U=\cos \varphi-\frac{\delta^{2} \operatorname{Ta} \sin \varphi}{2 \operatorname{Re}_{w}^{2}}\left[\exp \left(\operatorname{Re}_{w}\right)-\exp \left(\operatorname{Re}_{w} z\right)+\operatorname{Re}_{w}(z-1)\right] .
\end{gathered}
$$

Как видно из полученных решений (14), компоненты скорости $u$ и $V$ являются постоянными функциями.

Исследуем функцию $U$ на существование нулей на рассматриваемом промежутке $z \in[0 ; 1]$. Функция $U$ может принимать нулевое значение на нижней поверхности, заданной уравнением $z=0$, при выполнении следующего равенства, связывающего параметры граничных условий:

$$
\frac{2 \operatorname{Re}_{w}^{2} \operatorname{ctg} \varphi}{\operatorname{Ta} \delta^{2}}-\exp \left(\operatorname{Re}_{w}\right)+\operatorname{Re}_{w}=-1
$$

На верхней поверхности компонента скорости $U$ принимает нулевое значение при $\varphi=\pi / 2+\pi n(n-$ целое число).

Для исследования функции на интервале $z \in(0 ; 1)$ записываем уравнение:

$$
U=\cos \varphi-\frac{\operatorname{Ta}^{2} \sin \varphi}{2 \operatorname{Re}_{w}^{2}}\left[\exp \left(\operatorname{Re}_{w}\right)-\exp \left(\operatorname{Re}_{w} z\right)+\operatorname{Re}_{w}(z-1)\right]=0
$$

Преобразуем равенство (15), разделив все его слагаемые на коэффициент $\frac{\mathrm{Ta}^{2} \sin \varphi}{2 \mathrm{Re}_{w}^{2}}$, получим следующее уравнение:

$$
\exp \left(\operatorname{Re}_{w} z\right)-\operatorname{Re}_{w} z+\frac{2 \operatorname{Re}_{w}^{2} \cos \varphi}{\operatorname{Ta}^{2} \sin \varphi}-\exp \left(\operatorname{Re}_{w}\right)+\operatorname{Re}_{w}=0
$$

Исследование количества корней уравнения $U=0$ на интервале $(0 ; 1)$ сведем к исследованию равенства некоторых функций $f_{1}$ и $f_{2}$ :

$$
\exp \left(A_{1} z\right)=A_{1} z+A_{2}
$$

где $f_{1}=\exp \left(A_{1} z\right)$ (кривая 1, рис. 1), $f_{2}=A_{1} z+A_{2}$ (кривые 2-5, рис. 1), $A_{1}=\operatorname{Re}_{w}$, $\mathrm{A}_{2}=\exp \left(\operatorname{Re}_{w}\right)-\frac{2 \operatorname{Re}_{w}^{2} \cos \varphi}{\operatorname{Ta}^{2} \sin \varphi}-\operatorname{Re}_{w}$. Заметим, что $\left.f_{1}^{\prime}\right|_{z=0}=\left.f_{2}^{\prime}\right|_{z=0}$, т. е. угол наклона касательной функции $f_{1}$ в начале рассматриваемого отрезка $[0 ; 1]$ совпадает с функцией $f_{2}$ с точностью до константы. Как видно на графике (рис. 1), функции $f_{1}$ и $f_{2}$ могут пересекаться в одной 
точке или не иметь общих точек на рассматриваемом отрезке. Вторая точка пересечения ни в каком случае не будет принадлежать рассматриваемому отрезку.

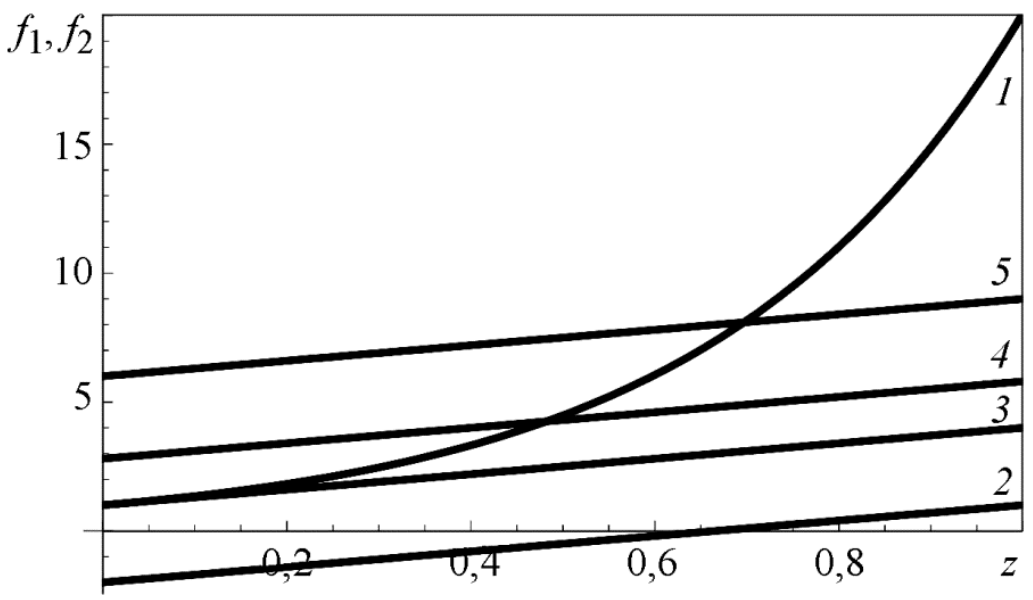

Рис. 1. Графическая интерпретация корней уравнения $U=0$ при условии идеального скольжения на нижней границе

Можно сделать заключение, что на интервале $z \in(0 ; 1)$ у функции $U$ может существовать не более одного корня при выполнении граничных условий (11), (12). А с учетом того, что остальные компоненты скорости $u$ и $V$ в данном случае являются постоянными функциями, можно сделать заключение, что при выполнении условия идеального скольжения на нижней границе и условий (12) на верхней границе в рассматриваемом слое вязкой несжимаемой жидкости может существовать не более одной застойной точки.

На рис. 2 изображены линии тока для случая задания условия идеального скольжения на нижней границе. Изменение направления движения жидкости наглядно показывает существование застойных точек.

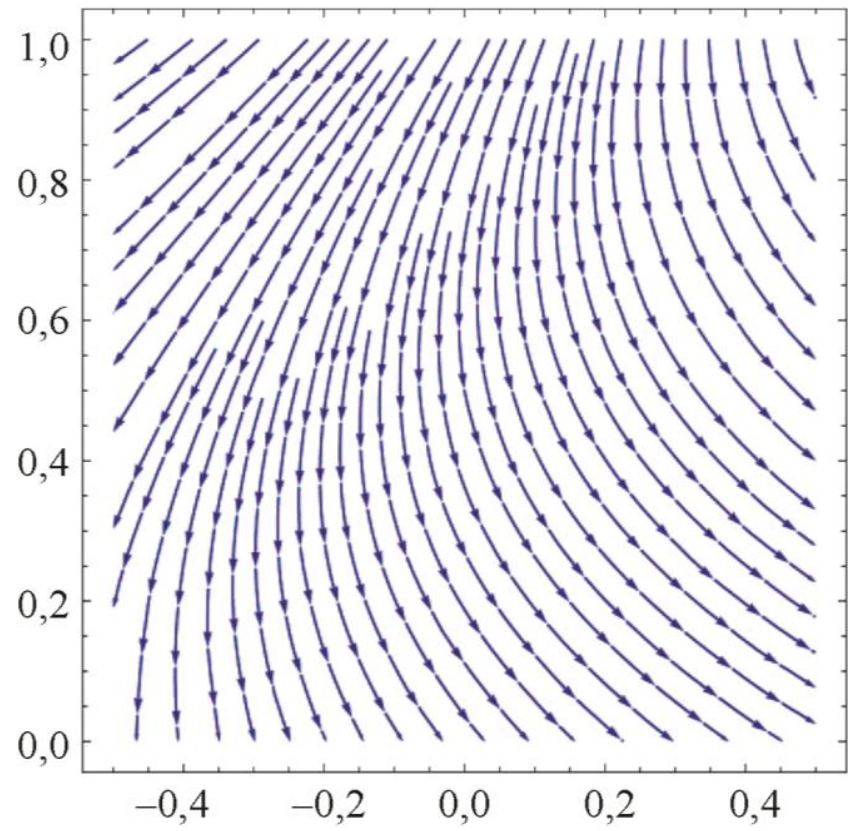

Рис. 2. Линии тока при $\delta=0,01 ; \varphi=-2 \pi / 3 ; \operatorname{Re}_{U}=1 \cdot 10^{5} ; \operatorname{Re}_{w}=-10 ; \mathrm{Ta}=3 \cdot 10^{5}$ 
На рис. 3 показана локализация корней функции $U$ на интервале $z \in(0 ; 1)$ при различных значениях модифицированного числа Тейлора Та. По физическому смыслу числа Тейлора получаем, что при значительном возрастании центробежных сил по сравнению с силами вязкого трения, нулевое значение функции $U$ будет локализоваться вблизи верхней границы.

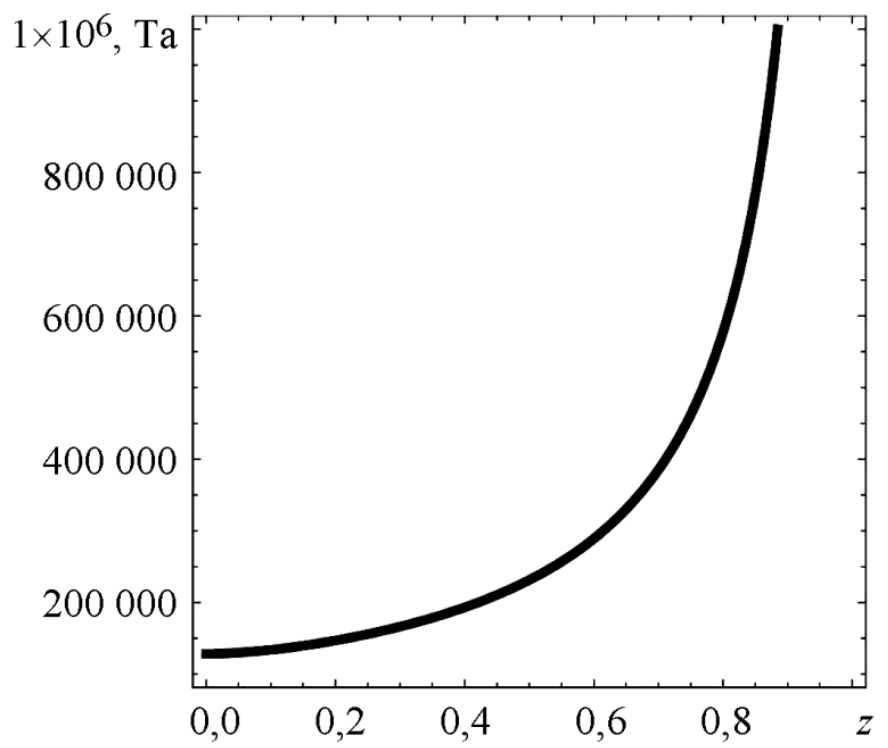

Рис. 3. Локализация корней $U$ для значений модифицированного числа Тейлора Та $\in\left[10^{5} ; 10^{6}\right]$ при $\delta=0,01 ; \varphi=-2 \pi / 3 ; \operatorname{Re}_{w}=-10$

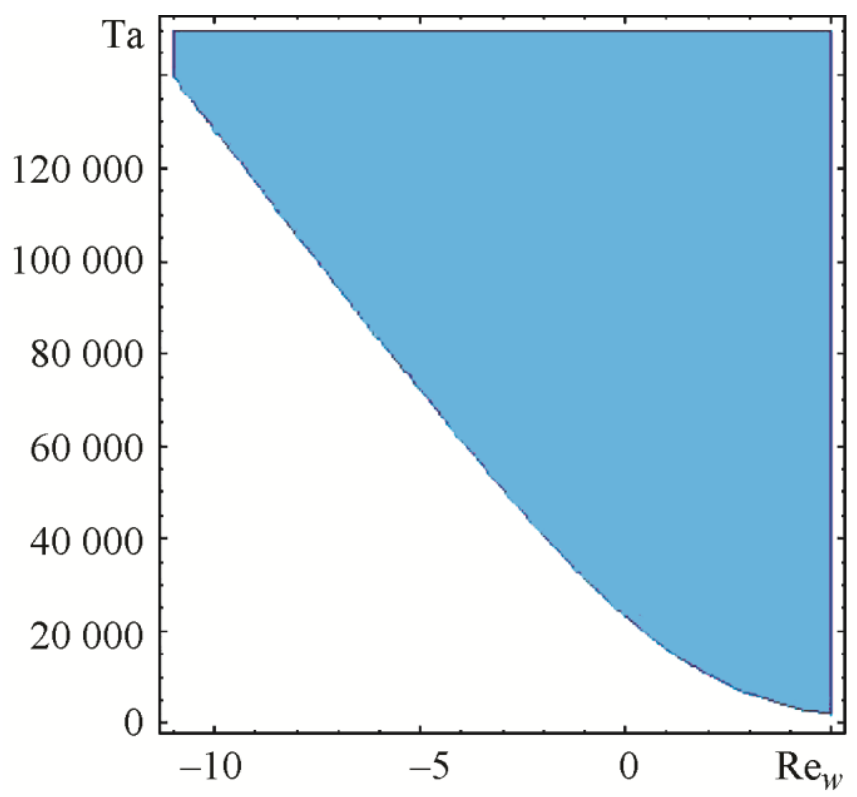

Рис. 4. Область существование точек противотечения функции $U$ при фиксированном $\varphi=-\frac{2 \pi}{3}$ и значениях параметров $\operatorname{Re}_{w} \in[-11 ; 5]$ и $\mathrm{Ta} \in\left[10^{3} ; 15 \cdot 10^{4}\right]$ 
Дополнительное исследование показывает существование точек противотечения компоненты скорости $U$ при различных значениях угла $\varphi$ и параметров подобия $\operatorname{Re}_{w}$ и Ta Например, при заданном значении угла $\varphi=-2 \pi / 3$ получаем область существования корней, изображенную на рис. 4. Рассматривались следующие значения параметров подобия: $\operatorname{Re}_{w} \in[-11 ; 5] ;$ Ta $\in[1000 ; 150000]$. На рис. 5 изображены области существования точек противотечения компоненты скорости $U$ при фиксированном числе Рейнольдса $\operatorname{Re}_{w}=-1$ и различных углах $\varphi \in[0 ; 2 \pi]$ и модифицированного числа Тэйлора Та $\in[1000 ; 200000]$.

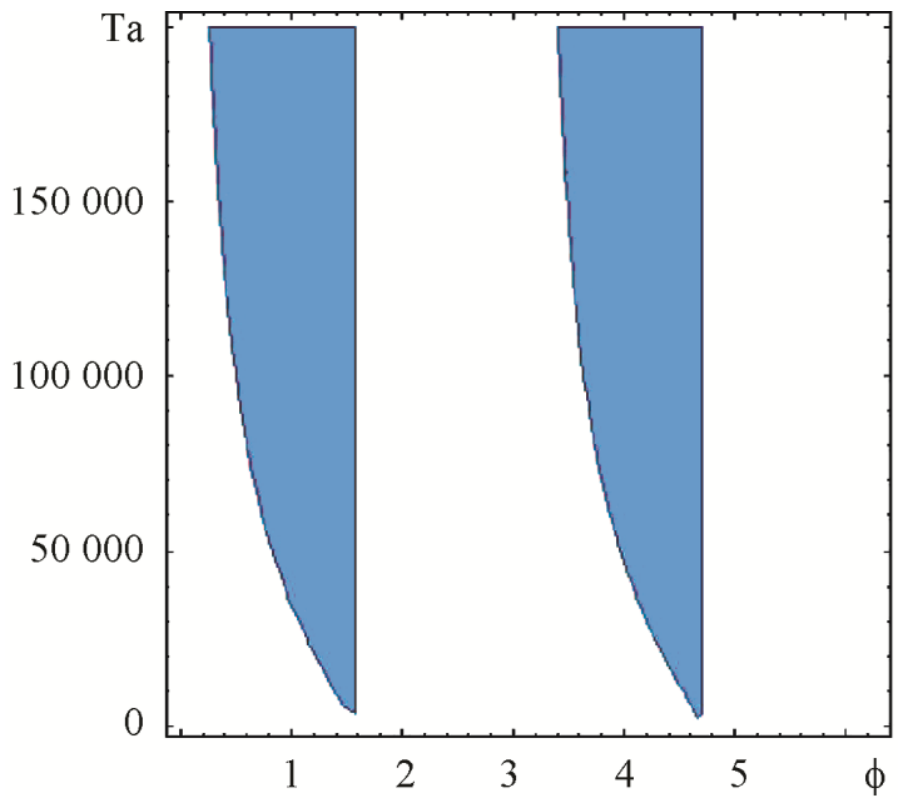

Рис. 5. Существование точек противотечения функции $U$ при фиксированном $\operatorname{Re}_{w}=-1$

$$
\text { и значениях } \varphi \in[0 ; 2 \pi] \text { и Та } \in\left[10^{3} ; 2 \cdot 10^{5}\right]
$$

\section{5. Заключение}

В статье получено обобщение установившегося классического течения Куэтта для трехмерных нелинейных вязких несжимаемых жидкостей. Показано, что в изобарическом течении при рассмотрении точных решений из класса линейно растущих скоростей по горизонтальным координатам с учетом граничного условия идеального скольжения возможно возникновение противотечения в слое жидкости. Показана локализация расслоения в приграничных слоях течения жидкости. Проведенный анализ решений применим для крупномасштабных течений Мирового океана.

\section{Литература}

1. Овсянников Л. В. Групповой анализ дифференциальных уравнений. - М. : Наука, 1978. - $400 \mathrm{c}$.

2. Применение теоретико-групповых методов в гидродинамике / В. К. Андреев, О. В. Капцов, В. В. Пухначев, А. А. Родионов. - Новосибирск : Наука, 1994. - 320 с.

3. Aristov S. N., Knyazev D. V., Polyanin A. D. Exact solutions of the Navier-Stokes equations with the linear dependence of velocity components on two space variables // Theor. Found. Chem. Eng. - 2009. - Vol. 43, no. 5. - P. 642-662. - DOI: 10.1134/S0040579509050066.

4. Аристов С. Н., Просвиряков Е. Ю. Неоднородные течения Куэтта // Нелинейная динамика. - 2014. - Т. 10, № 2. - C. 177-182. - DOI: 10.20537/nd1402004. 
5. Аристов С. Н., Просвиряков Е. Ю. Волны Стокса в завихренной жидкости // Нелинейная динамика. - 2014. - Т. 10, № 3. - C. 309-318. - DOI: 10.20537/nd1403005.

6. Aristov S. N., Prosviryakov E. Yu. A new class of exact solutions for three-dimensional thermal diffusion equations // Theor. Found. Chem. Eng. - 2016. - Vol. 50, no. 3. - P. 286-293. DOI: $10.1134 / \mathrm{S} 0040579516030027$.

7. Prosviryakov E. Yu. New Class of Exact Solutions of Navier-Stokes Equations with Exponential Dependence of Velocity on Two Spatial Coordinates // Theor. Found. Chem. Eng. - 2019. Vol. 53, no. 1. - P. 107-114. - DOI: 10.1134/S0040579518060088.

8. Lin C. C. Note on a class of exact solutions in magneto-hydrodynamics // Arch. Rational Mech. Anal. - 1957. - Vol. 1. - P. 391-395. - DOI: 10.1007/BF00298016.

9. Сидоров А. Ф. Об одном классе решений уравнений газовой динамики и естественной конвекции // Численные и аналитические методы решения задач механики сплошной среды. Свердловск : УНЦ АН СССР, 1981. - С. 101-117.

10. Sidorov A. F. Two classes of solutions of the fluid and gas mechanics equations and their connection to traveling wave theory // J. Appl. Mech. Tech. Phys. - 1989. - Vol. 30, no. 2. P. 197-203. - DOI: 10.1007/BF00852164.

11. Аристов С. Н., Шварц К. Г. Вихревые течения адвективной природы во вращающемся слое жидкости. - Пермь, 2006. - 155 с.

12. Аристов С. Н., Шварц К. Г. Вихревые течения в тонких слоях жидкости. - Киров : ВятГУ, 2011. - $207 \mathrm{c}$.

13. Андреев В. К. Решения Бириха уравнений конвекции и некоторые его обобщения : препринт № 1-10. - Красноярск : ИВМ СО РАН, 2010. - 68 с.

14. Andreev V. K., Bekezhanova V. B. Stability of nonisothermal fluids (Review) // J. Appl. Mech. Tech. Phys. - 2013. - Vol. 54, no. 2. - P. 171-184. - DOI: 10.1134/S0021894413020016.

15. Andreev V. K., Stepanova I. V. Unidirectional flows of binary mixtures within the framework of the Oberbeck-Boussinesq model // Fluid Dyn. - 2016. - Vol. 51, no. 2. - P. 136-147. DOI: $10.1134 / \mathrm{S} 0015462816020022$.

16. Modening of the convective fluid flows with evaporation in the two-layer system / O. N. Goncharova, M. Hennenberg, E. V. Rezanova, O. A. Kabov // Interfacial Phenomena and Heat Transfer. - 2013. - Vol. 1, no. 4. - P. 317-338. - DOI: 10.1615/InterfacPhenomHeatTransfer.v1.i4.20.

17. Aristov S. N., Knyazev D. V., Polyanin A. D. Exact solutions of the Navier-Stokes equations with the linear dependence of velocity components on two space variables // Theor. Found. Chem. Eng. - 2009. - Vol. 43, no. 5. - P. 642-662. - DOI: 10.1134/S0040579509050066.

18. Бетяев С. К. Асимптотические методы классической динамики жидкости. - МоскваИжевск : Институт компьютерных исследований, 2014. - 516 с.

19. Andreev V. K. Influence of the interfacial internal energy on the thermocapillary steady flow // Журнал Сибирского федерального университета. Серия : Математика и физика. - 2017. T. 10, № 4. - C. 537-547. - DOI: 10.17516/1997-1397-2017-10-4-537-547.

20. Неклассические модели конвекции: точные решения и их устойчивость / В. К. Андреев, В. Б. Бекежанова, М. В. Ефимова, И. И. Рыжков, И. В. Степанова // Вычислительные технологии. - 2009. - Т. 14, № 6. - С. 5-18.

21. Устойчивость двухслойных течений жидкости с испарением на границе раздела / В. Б. Бекежанова, О. Н. Гончарова, Е. В. Резанова, И. А. Шефер // Известия Российской академии наук. Механика жидкости и газа. - 2017. - № 2. - С. 23-35. - DOI: 10.7868/S0568528117020062.

22. Изучение конвективных течений жидкости и спутного потока газа с учетом испарения / О. Н. Гончарова, Е. В. Резанова, Ю. В. Люлин, О. А. Кабов // Теплофизика высоких температур. - 2017. - Т. 55, № 6. - C. 720-732. - DOI: 10.7868/S0040364417060072.

23. Аристов С. Н., Просвиряков Е. Ю. Крупномасштабные течения завихренной вязкой несжимаемой жидкости // Известия высших учебных заведений. Авиационная техника. 2015. - № 4. - С. 50. 
24. Аристов С. Н., Просвиряков Е. Ю. Нестационарные слоистые течения завихренной жидкости // Известия Российской академии наук. Механика жидкости и газа. - 2016. - № 2. C. 25 .

25. Аристов С. Н., Привалова В. В., Просвиряков Е. Ю. Стационарное неизотермическое течение Куэтта. Квадратичный нагрев верхней границы слоя жидкости // Нелинейная динамика. - 2016. - Т. 12, № 2. - C. 167-178. - DOI: 10.20537/nd1602001.

26. Привалова В. В., Просвиряков Е. Ю. Стационарное конвективное течение КуэттаХименца при квадратичном нагреве нижней границы слоя жидкости // Нелинейная динамика. - 2018. - Т. 14, № 1. - С. 69-79. - DOI: 10.20537/nd1801007.

27. Привалова В. В., Просвиряков Е. Ю. Точные решения Куэтта-Хименца для описания установившегося ползущего конвективного течения вязкой несжимаемой жидкости с учетом теплообмена // Вестник Самарского государственного технического университета. Серия Физико-математические науки. - 2018. - Т. 22, № 3. - С. 532-548. - DOI: 10.14498/vsgtu1638. 28. Vinogradova O. I., Belyaev A. V. Wetting, roughness and flow boundary conditions // J. Phys.: Condens. Matter. - 2011. - Vol. 23. - P. 184104. - DOI: 10.1088/0953-8984/23/18/184104.

29. Stone H., Stroock A., Ajdari A. Engineering flows in small devices: Microfluidics toward a lab-on-a-chip // Annual Review of Fluid Mechanics. - 2004. - Vol. 36. - P. 381-411. DOI: 10.1146/annurev.fluid.36.050802.122124.

30. Quere D. Wetting and roughness // Annu. Rev. Mater. Res. - 2008. - Vol. 38. - P. 71-99.

31. Whitesides G. M. The origins and the future of microfluidics // Nature. - 2006. - Vol. 442. P. 368-373. - DOI: 10.1038/nature05058.

32. Belyaev A. V., Vinogradova O. I. Effective slip in pressure-driven flow past superhydrophobic stripes // J. Fluid Mech. - 2010. - Vol. 652. - P. 489-499. - DOI: 10.1017/S0022112010000741.

33. Ландау Л. Д., Лифшиц Е. М. Гидродинамика. - 6-е. изд. - Москва : Физматлит, 2006. - 736 с.

34. Педлоски Дж. Геофизическая гидродинамика : в 2 т. - М. : Мир, 1984.

35. Vinogradova O. I. Drainage of a thin liquid film confined between hydrophobic surfaces // Langmuir. - 1995. - Vol. 11, iss. 6. - P. 2213-2220. - DOI: 10.1021/la00006a059.

36. Mehdizadeh A., Oberlack M. Analytical and numerical investigations of laminar and turbulent Poiseuille-Ekman flow at different rotation rates // Physics of Fluids. - Vol. 22, no. 10. P. 105104. - DOI: 10.1063/1.3488039.

37. Коротаев Г. К., Михайлова Э. Н., Шапиро Н. Б. Теория экваториальных противотечений в Мировом океане. - Киев : Наук. думка, 1986. - 208 с. 